\title{
Evaluación de la implantación de la Gamificación como metodología activa en la Educación Secundaria española
}

\author{
Julián Roa González - UDIMA \\ Almudena Sánchez Sánchez - UDIMA \\ Natalia Sánchez Sánchez - UDIMA
}

\author{
(1) 0000-0002-4017-3067 \\ (D) 0000-0002-4246-4132 \\ (i) 0000-0003-1937-3600
}

Recepción: 30.09.2020 | Aceptado: 05.02.2021

Correspondencia a través de ORCID: Julián Roa González

iD 0000-0002-4017-3067

Citar: Roa González, J., Sánchez Sánchez, A. y Sánchez Sánchez, N. (2021). Evaluación de la implantación de la Gamificación como metodología activa en la Educación Secundaria española. REIDOCREA, 10(12), $1-9$.

Resumen: Las metodologías activas han sido objeto de evaluación por el OIE de la UDIMA y se consideran un elemento indispensable para la innovación educativa. En este grupo de metodologías, la gamificación ha recibido mucha atención por parte de la comunidad académica y los medios de comunicación, destacando sus posibilidades y ventajas como elemento dinamizador de los procesos de enseñanzaaprendizaje, así como la mejora de la motivación en el alumnado. Este artículo persigue analizar, cuantitativamente, el porcentaje de uso de la gamificación en los centros de secundaria con currículum español y detectar variables que influyan en la implantación y empleo de esta metodología. Para realizarlo, se ha recogido una muestra no estratificada a través de cuestionarios, lo que ha permitido analizar las metodologías empleadas en las asignaturas de Lengua Castellana, Matemáticas, Geografía e Historia y Lengua Extranjera (inglés) durante el primer semestre del curso 2019-2020 en 165 centros educativos. De manera general, podemos decir que los datos recogidos y analizados en nuestro estudio sugieren que la gamificación no está suficientemente asentada en las instituciones educativas de secundaria con currículum español, aunque se observan diferencias importantes dependiendo de la titularidad del centro, así como el tipo de asignatura que se imparte.

Palabra clave: Innovación educacional

\section{Evaluation of the implementation of Gamification as an active methodology in Spanish Secondary Education}

\begin{abstract}
The active methodologies have been evaluated by the Observatory of Educational Innovation of the Distance University of Madrid and are considered an indispensable element for educational innovation. Within this group of methodologies, gamification has received a lot of attention from the academic community and the media, highlighting its possibilities and advantages as a dynamic element of the teaching-learning processes, as well as the improvement of motivation in the student body. This article aims to quantitatively analyze the percentage of use of gamification in secondary schools that follow the Spanish curriculum and detect variables that influence the implementation and use of this active methodology. To carry it out, a nonstratified sample has been collected through questionnaires, which has made it possible to analyze the methodologies used in the subjects of Spanish Language and Literature, Mathematics, Geography and History and Foreign Language (English) during the first semester of the 2019-2020 academic year in 165 educational centers. In general, we can say that the data collected and analyzed in our study suggest that gamification is not sufficiently established in secondary educational institutions that follow the Spanish curriculum, although important differences are observed depending on the ownership of the center, as well as the type of subject taught.
\end{abstract}

Keyword: Educational innovations

\section{Introducción}

Desde el comienzo del siglo XXI se ha venido cuestionando el papel que deberían tener el profesor y el estudiante para responder a los cambios sociales derivados de la sociedad de la información. Prensky (2001) estableció 18 características por las que los nativos digitales eran diferentes al resto de generaciones. Aquí nos centraremos en 
algunas de ellas, teniendo en cuenta las características del nivel educativo y el tema que nos ocupa:

- Se comunican de forma diferente con los demás y no necesitan conocer a sus interlocutores. Se identifican mediante apodos y avatares.

- El ocio es digital, ya que juegan en línea con jugadores que incluso ni conocen.

- Aprenden de manera diferente a través de wikis y tutoriales. Pueden aprender algunas cosas solo observando vídeos en línea realizados por otros usuarios.

- Buscan a través de buscadores en línea. Cualquiera con acceso a Internet puede acceder a la cultura, utilizando cada vez menos libros y enciclopedias en papel. El usuario debe ser muy selectivo, pues en muchas ocasiones nos encontramos con fuentes de baja calidad.

- Analizan de manera más crítica las situaciones a las que se enfrentan y tienen la información al alcance de su mano de manera rápida.

- Necesitan una enseñanza basada en competencias que refuerce la motivación.

Estos planteamientos originales se han ido desarrollando con el tiempo (Olivares y González, 2016) y han sido recogidos en gran medida en la legislación educativa española. Según se indica en el Preámbulo de la Ley Orgánica 8/2013, de 9 de diciembre, para la mejora de la calidad educativa:

"El sistema educativo debe posibilitar tanto el aprendizaje de cosas distintas como la enseñanza de manera diferente, para poder satisfacer a unos alumnos y alumnas, que han ido cambiando con la sociedad. Las habilidades cognitivas, siendo imprescindibles, no son suficientes; es necesario adquirir desde edades tempranas competencias transversales". (p. 97860).

Las escuelas deben, por tanto, adaptarse para ofrecer a los alumnos esas competencias que necesitarán en el futuro.

Dentro de este contexto, encontramos referencias que vienen señalando ya desde el año 2011 una nueva metodología que se define como Gamificación (Deterding et al., 2011; Kapp, 2012; Marczewski, 2013; Werbach y Hunter, 2012; Zichermann y Cunningham, 2011). En general, podemos afirmar que las definiciones coinciden en señalar que se utilizan mecánicas del juego en contextos de la vida cotidiana para influir en las personas que las utilizan.

Estas primeras definiciones se han matizado posteriormente para diferenciar la gamificación del aprendizaje basado en juegos y de los serious game. En Marczewski (2015) y Ordás (2018) podemos ver que, aunque los tres conceptos forman parte de los estudios sobre el pensamiento lúdico, existen diferencias importantes entre ellos. Cuando creamos un videojuego específico con un fin no lúdico, estamos ante un serious game. En cambio, cuando utilizamos un juego ya existente como complemento de la enseñanza, nos encontramos ante un aprendizaje basado en juegos. A diferencia de estos dos planteamientos, la gamificación incorpora algunos elementos del juego para aprender.

En paralelo a esta concreción y diferenciación, se han establecido teóricamente los elementos que utilizan los juegos. Estos son: dinámicas, mecánicas y componentes (Alejaldre y García, 2015; Werbach y Hunter, 2015; Wiklund y Wakerius, 2016). Las dinámicas son necesidades e inquietudes humanas que determinan el comportamiento y que se relacionan, fundamentalmente, con la motivación. No se pueden plasmar de forma tangible, pero son fundamentales para el éxito de un juego. Las mecánicas son los componentes que regulan el juego y establecen su funcionamiento. Sirven, 
fundamentalmente, para impulsar la acción. Y por último, los componentes, son elementos de puntuación o anotación, como las insignias, los combates o las clasificaciones que sirven para para establecer una dinámica positiva cumpliendo con la mecánica del juego.

En los últimos 5 años, numerosos estudios (Amezcua y Amezcua, 2018; Díaz, 2017; Ortiz, 2017; Gallego y Ágredo, 2016; García, 2016; González et al., 2017; Pérez, Rivera y Trigueros, 2017; Quintanal, 2016) han dado cuenta de experiencias concretas de aula en España. Adicionalmente, varios autores como Zichermann y Cunningham (2011); Kapp (2012) o Bruder (2015) han establecido las principales ventajas derivadas del uso de esta metodología, entre las que destacan las siguientes:

- El incremento de la motivación en el alumnado, pues al tener un objetivo o meta, se involucran más en la dinámica de aprendizaje y, superar el reto, los lleva a un sentimiento de logro y éxito.

- Provee un ambiente seguro de aprendizaje animando al alumnado a realizar nuevos retos, a investigar nuevas soluciones.

- El alumnado es consciente de su progreso, gracias a la retroalimentación que se le proporciona para guiarle hacia el resultado correcto.

- Genera cooperación, debido a que se suele trabajar en equipo para lograr el objetivo.

- Mejora el autoconocimiento sobre las capacidades que se poseen, ya que el juego le permite comprobar sus habilidades y destrezas.

- Favorece la retención del conocimiento adquirido de forma significativa.

Debemos tener en cuenta que el empleo de la gamificación también tiene inconvenientes, como señala Pérez de Villaamil (2018):

- Elevado coste, debido a la producción de material audiovisual educativo utilizado, a las herramientas educativas utilizadas y a la renovación del material.

- Elevado tiempo de elaboración del material.

- Distracción con el juego, con lo que implica perder tiempo.

- Dificultad para desarrollar la habilidad de la expresión oral.

- Dificultad para conseguir un equilibrio entre el carácter lúdico y formativo.

- Desmotivación una vez hayan superado la novedad inicial.

A pesar del desarrollo y de la atención recibida por la comunidad docente y académica, en nuestro país no se han realizado aún estudios que evalúen el grado de implantación de la gamificación en la Enseñanza Secundaria Obligatoria y el Bachillerato. Por ese motivo, el presente estudio analiza los datos recogidos en una encuesta realizada a estudiantes del Máster en Formación del Profesorado durante su periodo de prácticas, lo que ha permitido analizar la implantación observada de esta metodología en 165 centros educativos durante el curso 2019-2020.

\section{Objetivos o hipótesis}

La investigación se ha centrado en conocer la situación actual en la educación secundaria española en torno a la gamificación. De forma concreta, se busca dar respuesta a los siguientes objetivos:

- Analizar y proporcionar datos cuantitativos acerca del uso de la gamificación como metodología en centros de educación secundaria que siguen currículum español. 
- Detectar variables que influyan significativamente en la implantación y utilización de la gamificación como metodología activa innovadora.

\section{Métodos}

El estudio se llevó a cabo entre octubre de 2019 y enero de 2020, se obtuvieron 165 respuestas de estudiantes del Máster Universitario en Formación del Profesorado de Educación Secundaria que habían realizado sus prácticas presenciales en centros educativos con currículum español en los niveles educativos de Educación Secundaria Obligatoria, Bachillerato, Formación Profesional y Enseñanza Oficial de Idiomas. Se analizaron las respuestas de $n=165$ estudiantes $(64,84 \%$ mujeres, $35,15 \%$ hombres, edad media $=32,34$ años). No fue necesario excluir ninguna respuesta, pues todos los cuestionarios recogidos incluían todos los apartados y habían sido cumplimentados de forma adecuada. Los participantes del estudio pertenecen a cuatro especialidades diferentes dentro de los estudios de Máster (45,45\% Matemáticas, 23,03\% Geografía e Historia, 21,81\% Lengua extranjera y 9,69\% Lengua Castellana).

Los datos se recogieron mediante un cuestionario informatizado que compilaba datos estadísticos generales e información específica sobre la innovación educativa observada durante su estancia de prácticas en centros de educación secundaria.

El cuestionario ha sido elaborado por los profesores del Observatorio de Innovación Educativa de la Universidad a Distancia de Madrid y cuenta con 30 ítems, pudiendo cumplimentarse en, aproximadamente, 35 minutos.

Una vez recogidos los datos, se han seguido las siguientes fases para su codificación:

1. Unificación de las respuestas aportadas. La variable "objeto de estudio" presentaba 5 posibilidades de implantación que se han dicotomizado. Tras este proceso, se han obtenido las siguientes opciones de respuesta para la variable:

- Centro que no emplea la gamificación como metodología o lo hace de forma superficial.

- Centro que emplea la gamificación como metodología en un porcentaje de las sesiones superior al $25 \%$.

2. Análisis cuantitativo de los datos. Aplicando técnicas básicas de estadística descriptiva, en particular, tablas de contingencia, se han obtenido los diferentes grados de implantación de la metodología, teniendo en cuenta las opciones de respuesta proporcionadas en la fase anterior.

\section{Resultados}

Como resultado de la investigación realizada sobre el grado de implantación de la gamificación en los centros de educación secundaria con currículum español, presentamos a continuación las siguientes tablas de resultados. 
Tabla 1. Grado de implantación de la gamificación en porcentaje y número de centros sobre el total analizado.

Tipologías de aula $\%$ de centros (.$^{\circ} \stackrel{0}{\text { de }}$ centros $)$

Centro que no emplea la gamificación como metodología o lo

hace de forma superficial

$144(87,27 \%)$

Centro que emplea la gamificación como metodología en un porcentaje de las sesiones superior al $25 \%$

$21(12,72 \%)$

Total general

$100,00 \%(165)$

De acuerdo a los resultados presentados en la Tabla 1, señalar que a pesar del desarrollo de los últimos años, la gamificación solo se emplea como metodología destacada en el $12,72 \%$.

A continuación, se ha realizado un desglose de la Tabla 1 con el fin de determinar, según la titularidad del centro, los porcentajes de implantación de los diferentes entornos en el aula. En la Tabla 2 se muestran los resultados obtenidos para la enseñanza privada; en la Tabla 3, para la enseñanza concertada, y en la tabla 4, para la enseñanza pública.

Tabla 2. Grado de implantación de la gamificación en la enseñanza privada en porcentaje y número de centros sobre el total analizado.

Tipologías de aula $\%$ de centros (n. de centros)

Centro que no emplea la gamificación como metodología o lo hace de forma superficial

Centro que emplea la gamificación como metodología en un porcentaje de las sesiones superior al $25 \%$

$14,28 \%(5)$

Total general $100,00 \%(35)$

Tabla 3. Grado de implantación de la gamificación en la enseñanza concertada en porcentaje y número de centros sobre el total analizado.

Tipologías de aula

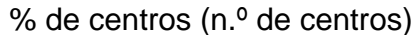

Centro que no emplea la gamificación como metodología o lo

hace de forma superficial

$84,21 \%(80)$

Centro que emplea la gamificación como metodología en un porcentaje de las sesiones superior al $25 \%$

$15,78 \%(15)$

Total general $100,00 \%(95)$

Tabla 4. Grado de implantación de la gamificación en la enseñanza concertada en porcentaje y número de centros sobre el total analizado.

Tipologías de aula $\%$ de centros ( $\mathrm{n} .^{\circ}$ de centros)

Centro que no emplea la gamificación como metodología o lo hace de forma superficial

Centro que emplea la gamificación como metodología en un porcentaje de las sesiones superior al $25 \%$

Total general $100,00 \%(35)$

En relación con los resultados presentados en las Tablas 2, 3 y 4, resaltar que el uso de la gamificación no es una metodología dominante en ninguna de las titularidades de centro exploradas. Sin embargo, parece que en la enseñanza privada y concertada hay una tendencia a utilizar esta metodología en cerca del 15\% de los centros, mientras que en la enseñanza pública sigue siendo una metodología casi irrelevante en los centros estudiados. 
Adicionalmente, se ha realizado un desglose de la Tabla 1 con el fin de determinar si existen diferencias en función de la asignatura que se estaba impartiendo. En la Tabla 5 se muestran los resultados obtenidos para las materias de matemáticas, lengua y literatura castellana, inglés y geografía e historia.

Tabla 5. Grado de implantación de la gamificación por asignatura en porcentaje y número de centros sobre el total analizado.

\begin{tabular}{|c|c|c|c|}
\hline Especialidad & $\begin{array}{l}\text { Centro que no emplea la } \\
\text { gamificación como } \\
\text { metodología o lo hace de } \\
\text { forma superficial }\end{array}$ & $\begin{array}{c}\text { Centro que emplea la } \\
\text { gamificación como } \\
\text { metodología en un } \\
\text { porcentaje de las sesiones } \\
\text { superior al } 25 \%\end{array}$ & $\begin{array}{c}\text { Total } \\
\text { general }\end{array}$ \\
\hline Matemáticas & $91 \%(68)$ & $9 \%(7)$ & $100,00 \%$ \\
\hline $\begin{array}{l}\text { Lengua y Literatura } \\
\text { Castellana y Lengua } \\
\text { extranjera Inglés }\end{array}$ & $83 \%(43)$ & $17 \%(9)$ & $100,00 \%$ \\
\hline Geografía e Historia & $87 \%(33)$ & $13 \%(5)$ & $100,00 \%$ \\
\hline
\end{tabular}

La Tabla 5 nos permite ver que las asignaturas relacionadas con las lenguas son más proclives a emplear la gamificación (17\%) que las asignaturas de matemáticas (9\%).

Cabe destacar que, aunque la metodología de gamificación se usa en cerca de un 12\% de los centros, su uso por encima del $50 \%$ de las sesiones solo se ha observado en el $3 \%$ de los centros analizados.

\section{Discusión}

Dentro del campo de la innovación educativa existen múltiples aspectos que deben tenerse en cuenta: la organización del tiempo y del espacio, los recursos tecnológicos y manipulativos o el tipo y enfoque de evaluación que se lleva a cabo (Cabero Almenara, 2015). Además de estos elementos, la metodología es un factor determinante a la hora de evaluar la innovación educativa y en los últimos años se está trabajando intensamente en el concepto de metodologías activas como un medio para mejorar el aprendizaje de modo que el alumnado sea protagonista del proceso, se pueda autoregular y aumente su motivación. Bajo este paraguas, podemos encontrar algunas como el aprendizaje cooperativo, el aprendizaje basado en proyectos y basado en problemas, la gamificación, el aprendizaje y servicio o la clase invertida (flipped classroom).

Este trabajo se centra en el estudio de la gamificación como metodología activa e innovadora basado, fundamentalmente, en dos indicadores:

- Su novedad dentro del panorama educativo, datando el primer empleo del término de 2008 (Deterding et al., 2011).

- La amplia difusión de trabajos con resultados prometedores en torno a esta metodología (Amezcua y Amezcua, 2018).

Sin embargo, a pesar de los trabajos teóricos de definición (Bruder, 2015; Kapp, 2012; Zichermann y Cunningham, 2011) y de los trabajos sobre experiencias gamificadas, no existen estudios objetivos que evalúen el grado de implantación que está teniendo esta metodología en los centros educativos de educación secundaria que siguen el currículum español. Nos encontramos, por tanto, ante una metodología activa prometedora de la que conocemos experiencias positivas y de la que apenas se dispone 
de datos objetivos sobre su implantación, salvo afirmaciones generales sobre su desarrollo en los últimos años.

Este trabajo de investigación pretende contribuir a suplir esta carencia detectada, ofreciendo una primera visión de la situación actual en la educación secundaria española en torno a la gamificación. A diferencia de otros trabajos, en este caso se pretende aportar y analizar datos cuantitativos acerca del empleo de esta metodología en una muestra no estratificada de 165 centros. Este estudio, aunque limitado por el tamaño y la composición de la muestra, ha permitido observar la baja implantación que está teniendo esta metodología en los centros de Educación Secundaria Obligatoria, Bachillerato, Formación Profesional y Enseñanza Oficial de Idiomas que siguen el currículum español, además de que, entre las variables que parecen influir en su implantación, aparecen la titularidad de los centros y la asignatura en que se lleva a cabo esta metodología.

De manera general y de acuerdo con los resultados presentados en las Tablas 1, 2, 3 y 4 , cabe destacar que esta metodología se usa de una forma destacada en el $15 \%$ de los centros privados y concertados estudiados y en el $3 \%$ de los centros públicos. Este dato es preocupante en términos de equidad educativa e igualdad de oportunidades, ya que no todos los estudiantes del sistema están recibiendo las mismas oportunidades para alcanzar el aprendizaje, al menos no con los mismos métodos.

Adicionalmente a esta implantación, a nivel general se ha detectado que una variable importante parece ser la asignatura o materia en la que se aplica, llegando a valores superiores al $17 \%$ en la enseñanza de las lenguas (castellana e inglesa), en torno a un $13 \%$ en la enseñanza de la geografía y la historia y a valores inferiores al $10 \%$ en la enseñanza de las matemáticas. Atendiendo a las altas tasas de abandono y desmotivación que suelen presentar los alumnos que cursan la asignatura de matemáticas en educación secundaria (Ferrero y Oloriz, 2016) y los resultados que se obtienen en las pruebas internacionales de evaluación en esta materia como el TIMSS (Mullis, Martin y Michael, 2019), se puede inferir que una implementación de la gamificación en esta asignatura en niveles similares a las lenguas o la geografía y la historia, podría suponer una oportunidad para mejorar el rendimiento matemático.

Finalmente, observando estos primeros resultados, se plantea la necesidad de continuar investigando la potencialidad de esta metodología activa innovadora, divulgando sus beneficios a nivel social y formando a los profesores en activo y futuros profesores en su correcto empleo y planteamiento en todas las asignaturas del currículum español que se imparte en la educación secundaria.

\section{Referencias}

Alejaldre, L. y García, AL. (2015). Gamificar: el uso de los elementos del juego en la enseñanza de español. En M.P. Celma, M.J. Gómez, y C. Morán (Eds.), Actas del $L$ congreso internacional de la AEPE (73-83). Burgos: Editorial Universidad Isabel I de Castilla.

Amezcua, T. y Amezcua, P. (2018). La gamificación como estrategia de motivación en el aula. En A. Torres y L.M. Romero (Eds), Gamificación en Iberoamérica. Experiencias desde la Comunicación y la Educación (137-146). Ecuador: Editorial Universidad Politécnica Salesiana.

Bruder, P. (2015). Game on: Gamification in the Classroom. Education Digest, 80(7), 56-60. 
Cabero Almenara, J. (2015). Reflexiones educativas sobre las tecnologías de la información y la comunicación (TIC). Revista Tecnología, Ciencia y Educación, 1, 19-27.

Deterding, S., Dixon, D., Khaled, R., \& Nacke, L. (2011). From game design elements to gamefulness: Defining "gamification". En Memorias del 15th International Academic MindTrek Conference: Envisioning Future Media Environments (9-15). New York, NY: ACM.

Díaz, PP. (2017). Gamificando con Kahoot en evaluación formativa. Revista Infancia, Educación y Aprendizaje, 3(2), (112-117).

Ferrero, E. y Oloriz, M. (2016). Aplicación de estrategias motivacionales para mejorar la aprobación en matemáticas y disminuir el abandono. Congresos CLABES. Recuperado de https://revistas.utp.ac.pa/index.php/clabes/article/view/1395

Gallego Aguilar, AF. y Ágredo Ramos, AF. (2016). Implementando una metodología de gamificación para motivar la lectura y escritura en jóvenes universitarios. Kepes, №. 14, 61-81.

García, CM. (2016). La senda del maestro: experiencias de gamificación en el aula universitaria. Comunicación del XII "Congreso Español de Sociología. Grandes transformaciones sociales, nuevos desafíos para la sociología. Recuperado de http://fes-sociologia.com/files/congress/12/papers/4288.pdf

González Reyes, JA., Olivares Granados, SA., García Sánchez, E. y Figuero Melchor, IG. (2017). Propuesta de Gamificación en el aula: Uso de una plataforma para motivar a los estudiantes del Programa Académico de Informática de la Universidad Autónoma de Nayarit. Revista Educateconciencia, 14(13), 70-79.

Kapp, KM. (2012). The Gamification of Learning and Instruction: Game-Based Methods and Strategies for Training and Education. New York: Pfeiffer.

Ley Orgánica 8/2013, de 9 de diciembre (BOE del 10 de diciembre), para la mejora de la calidad educativa (LOMCE).

Marczewski, A. (2013). Gamification: a simple introduction. Andrzej Marczewski.

Marczewski, A. (2015). Even Ninja Monkeys Like to Play: Gamification, Game Thinking and Motivational Design. California: CreateSpace Independent Publishing Platform.

Mullis, I., Martin, VS., \& Michael, O. (2019): Trends in International Mathematics and Science Study 2019. Recuperado de https://www.iea.nl/publications/assessmentframework/timss-2019-assessment-frameworks

Olivares, S. y González, J. (2016). La generación Z y los retos del docente. En I. Velasco y M. Páez (eds.). Los retos de la docencia ante las nuevas características de los estudiantes universitarios (114-124). México: Editorial Universidad Autónoma de Nayarit.

Ordás, A. (2018). Gamificación en bibliotecas: El juego como inspiración. Barcelona: Ed. UOC. 
Ortiz Carpintero, T. (2017). Gamificación: La vuelta al mundo en 80 días. Revista Infancia, educación y aprendizaje (IYEA) 3(2), 397-403.

Pérez de Villaamil, T. (2018). Gamificación en el aula: ventajas y desventajas. Recuperado de https://www.smartmind.net/blog/gamificacion-en-el-aulaventajas-y-desventajas/

Pérez López, IJ., Rivera García, E. y Trigueros Cervantes, C. (2017). La profecía de los elegidos: un ejemplo de gamificación aplicado a la docencia universitaria. Revista Internacional de Medicina y Ciencias de la Actividad Física y el Deporte, 17(66), 243-260.

Prensky, M. (2001). Digital natives, digital inmigrants. On the Horizon, 9(5),1-6.

Quintanal Pérez, F. (2016). Aplicación de herramientas de gamificación en física y química de secundaria. Opción, 32(12), 327-348.

Werbach, K., \& Hunter, D. (2012). For the win: How game thinking can revolutionize your business. Philadelphia, PA: Wharton School Press.

Werbach, K., \& Hunter, D. (2015). The gamification toolkit: Dynamics, mechanics, and components for the win. Philadelphia, PA: Wharton School Press.

Wiklund, E., \& Wakerius, V. (2016). The Gamification Process: A framework on gamification. Recuperado de http://www.divaportal.org/smash/get/diva2:931932/FULLTEXT01.pdf

Zichermann, G., \& Cunningham, C. (2011). Gamification by design: Implementing game mechanics in web and mobile apps. Canada: O'Reilly Media. 